\title{
Les mécanismes de l'apprentissage stratégique dans les coentreprises internationales
}

\author{
Elie Virgile Chrysostome ${ }^{1}$ \\ Université de Moncton
}

\section{Introduction}

L'apprentissage constitue l'une des principales motivations justifiant la création d'une coentreprise. En effet, la coentreprise facilite l'acquisition de nouvelles connaissances et, de ce point de vue, constitue un cadre favorable pour le développement de compétences stratégiques. Toutefois, la coentreprise ne constitue pas une solution infaillible aux préoccupations d'acquisition de connaissances, car si dans certains cas l'apprentissage de savoirfaire peut effectivement avoir lieu de manière relativement bien réussie, dans de nombreux autres cas l'apprentissage espéré peut échouer pour toutes sortes de raisons dont les conflits de pouvoir, de culture ou d'intérêts. L'échec de l'apprentissage dans les coentreprises internationales n'est pas un fait surprenant puisqu'en général les coentreprises échouent beaucoup plus qu'elles ne réussissent.

L'apprentissage dans les coentreprises internationales représente un exercice difficile à cause de la distance institutionnelle, culturelle et géographique qui existe entre les pays d'où proviennent les entreprises-mères. L'objectif de cet article est donc de contribuer à une meilleure compréhension du phénomène d'apprentissage dans les coentreprises internationales. Il vise plus précisément à montrer comment l'apprentissage peut favoriser le développement de compétences stratégiques au sein de ces coentreprises. Pour ce faire, il s'articule autour de trois parties, à savoir l'acquisition des connaissances, la création conjointe des connaissances, deux modes fondamentaux d'apprentissage au sein des coentreprises internationales, et les conditions de succès d'un tel apprentissage.

\section{L'acquisition des connaissances}

L'acquisition de nouvelles connaissances au sein d'une coentreprise est essentiellement le fait des individus qui proviennent des entreprises-mères et qui s'engagent dans un processus de partage de leurs expériences. Dans un tel processus, l'histoire de l'organisation et dans le cas d'espèce celle de la coentreprise, ses valeurs et en particulier les apprentissages qu'elle a réalisés avec le temps jouent un rôle important. Cette histoire constitue le point de départ du développement de connaissances. L'acquisition de nouvelles connaissances au sein d'une coentreprise ne se fait pas automatiquement quoique la coentreprise constitue un contexte particulièrement favorable à la diffusion des connaissances.

En effet, l'acquisition de nouvelles connaissances au sein des coentreprises internationales requiert des conditions particulières qui sont évoquées dans le dernier volet de cet article. Néanmoins, d'autres facteurs comme la nationalité des entreprises-mères, la structure organisationnelle de la coentreprise, une définition claire de ses objectifs ainsi que son mode de contrôle sont autant d'éléments qui influencent l'acquisition des connaissances.

La coentreprise à un parent dominant est moins propice à l'acquisition de connaissances que la coentreprise à gestion partagée. Ceci

est particulièrement vrai lorsque le parent dominant dans la coentreprise est l'entreprise-mère locale, qui la plupart du temps cherche à acquérir les connaissances de son partenaire étranger. Évidemment, l'une des raisons qui prédispose plus facilement la coentreprise à gestion partagée à une acquisition de connaissances est le fait que les décisions qui y sont prises sont le résultat d'intenses négociations.

Ainsi par exemple, la coentreprise à un parent dominant est moins propice à l'acquisition de connaissances que la 
coentreprise à gestion partagée. Ceci est particulièrement vrai lorsque le parent dominant dans la coentreprise est l'entreprise-mère locale, qui la plupart du temps cherche à acquérir les connaissances de son partenaire étranger. Évidemment, l'une des raisons qui prédispose plus facilement la coentreprise à gestion partagée à une acquisition de connaissances est le fait que les décisions qui y sont prises sont le résultat d'intenses négociations. Cependant, l'apparition de conflits d'ordre culturel dans la coentreprise à gestion partagée affecte beaucoup plus facilement le processus d'acquisition de connaissances que dans la coentreprise à un parent dominant.

Il existe différents mécanismes d'acquisition de connaissances. Huber ${ }^{2}$ en a identifié cinq à partir desquels il a proposé les différents types d'acquisition de connaissances ci-après: l'acquisition par expérience, l'acquisition par mimétisme, l'acquisition de l'extérieur et l'acquisition par la recherche. Le cinquième type d'acquisition, à savoir l'acquisition par héritage, semble plus approprié aux filiales. Néanmoins, il existe deux mécanismes de base qui favorisent l'acquisition des connaissances en vue d'un développement de compétences stratégiques, notamment dans les coentreprises internationales: il s'agit du transfert et de l'internalisation des connaissances.

\section{Le transfert de connaissances}

Le transfert de connaissances joue un rôle important dans le développement de compétences stratégiques. En effet, le transfert de connaissances permet à la coentreprise d'avoir accès aux connaissances des entreprises-mères, notamment par partage d'expériences entre les employés détachés en son sein par les entreprises-mères.

De ce point de vue, le transfert permet à la coentreprise d'accumuler des connaissances qui lui permettent de développer de nouvelles habiletés qui peuvent se traduire par de meilleures performances pour la coentreprise. Ces connaissances peuvent être tacites ou explicites. Lorsqu'elles sont explicites, elles sont moins difficiles à imiter par les concurrents et de ce point de vue ne confèrent pas au partenaire apprenant des avantages compétitifs durables. Il est donc souhaitable pour la coentreprise que les connaissances qui lui sont transférées ne soient pas exclusivement des connaissances explicites, mais soient aussi des connaissances tacites. Ces dernières sont en effet difficiles à décoder par les concurrents et peuvent de ce point de vue conférer un caractère stratégique aux habiletés dont elles ont contribuées au développement.
Cependant, la question qui pourrait se poser est celle de savoir comment se fait concrètement le transfert des connaissances tacites, étant donné que contrairement aux connaissances explicites, elles n'existent pas sous une forme explicitement codifiée qui puisse les prédisposer au transfert. La réponse à cette question délicate renvoie aux deux types de transfert de connaissances tacites identifiés dans la littérature scientifique. Il s'agit d'une part du transfert par métaphores, et d'autre part du transfert sans langage. Le transfert par métaphores consiste à identifier et à comprendre le langage de métaphores dans lequel se trouvent emmagasinées les connaissances tacites du partenaire, puis à utiliser ce langage pour les acquérir. Le langage de métaphores dont il s'agit ici est bien entendu un langage qui est spécifique au partenaire, et qui de ce point de vue ne peut pas être décodé par des individus qui lui sont étrangers $^{3}$. L'utilisation d'un tel langage permet de convertir les connaissances tacites acquises en compétences stratégiques, tout en créant en même temps une ambiguïté causale qui les rend difficiles à imiter ${ }^{4}$. Le transfert sans langage quant à lui consiste en une acquisition des connaissances tacites du partenaire grâce à une observation et une imitation de ses pratiques. Il s'agit donc d'un partage d'expériences qui conduit à un développement d'habiletés spécifiques qui peuvent êtres transformées par la suite.

\section{L'internalisation des connaissances}

L'internalisation des connaissances constitue une composante fondamentale de l'apprentissage, et par ricochet une condition indispensable pour le développement de compétences stratégiques. En effet, l'internalisation permet à la coentreprise de s'approprier les connaissances de son partenaire, afin de pouvoir les déployer pour le développement de nouveaux produits ou de nouvelles opportunités d'affaires.

L'internalisation ne se résume pas à un simple accès aux connaissances des entreprises-mères par le canal d'un accord de licence, d'une sous-traitance ou encore d'une assistance technique ponctuelle du partenaire à l'occasion d'opérations critiques. Elle constitue plutôt un réel mécanisme d'appropriation effective des habiletés des entreprises-mères qui se traduit par le développement ou le renouvellement de la mémoire organisationnelle. Le développement ou le renouvellement de la mémoire organisationnelle (base de connaissances ) constitue pour la coentreprise un atout important dans le développement 
de compétences stratégiques. En effet, le développement ou le renouvellement de la mémoire organisationnelle permet à la coentreprise d'exploiter de nouvelles potentialités fondées sur une synergie entre ses propres connaissances et les connaissances transmises par les entreprises-mères.

\section{Le développement ou le renouvellement de la mémoire organisationnelle (base de connaissances) constitue pour la coentreprise un atout important dans le développement de compétences stratégiques. En effet, le développement ou le renouvellement de la mémoire organisationnelle permet à la coentreprise d'exploiter de nouvelles poten- tialités fondées sur une synergie entre ses propres connaissances et les connaissances transmises par les entreprises-mères.}

L'internalisation doit faire l'objet d'une intention clairement exprimée au partenaire, sinon elle peut être à l'origine de conflits. Le degré de l'intention d'internaliser les connaissances du partenaire dépend de la perception que l'entreprise a de ces connaissances, et notamment des avantages qu'elle peut en tirer. Ainsi, l'intention d'internaliser les connaissances du partenaire sera forte et clairement exprimée si l'entreprise les perçoit comme étant d'une importance cruciale pour sa croissance et d'une compétitivité durable.

\section{La création conjointe de connaissances}

La création conjointe de nouvelles connaissances constitue le deuxième mode fondamental d'apprentissage dans les coentreprises. Elle revêt une grande importance pour la vie de ces coentreprises puisque les connaissances développées constituent un actif fondamental qui influence leur performance, et sert aussi de base au maintien de leurs avantages compétitifs.

Malheureusement, le phénomène de la création des connaissances organisationnelles reste encore mal connu malgré toutes les recherches dont il fait l'objet. Il ne fait cependant aucun doute que les interactions sociales entre les membres de l'organisation jouent un rôle fondamental dans la création de nouvelles connaissances. Plus spécifiquement, dans les coentreprises où ces interactions entre les individus provenant des entreprises-mères constituent un important mécanisme de l'apprentissage, elles permettent un échange d'expériences qui génère un développement de nouvelles connaissances. Ces individus constituent en d'autres termes une communauté d'interactions au sein de laquelle a lieu un partage de connaissances qui contribue à une création conjointe de connaissances. Les interactions sociales qui conduisent à une création de nouvelles connaissances ont lieu dans le cadre d'expérimentations conjointes au sein des coentreprises. Cependant, de nouvelles interprétations conjointes de connaissances existantes constituent également un mécanisme de création de nouvelles connaissances dans les coentreprises.

Les expérimentations conjointes se font à travers la réalisation de diverses expériences conjointes de type opportuniste. Il s'agit d'expériences destinées à remédier à des problèmes de la coentreprise, et au cours desquelles les individus provenant des différentes entreprises-mères interagissent les uns avec les autres en partageant leurs expériences passées. Ce faisant, ils disséminent à différents niveaux opérationnels de la coentreprise des connaissances qui sont par la suite internalisées et amplifiées au sein de cette dernière, ce qui donne lieu à une création de nouvelles connaissances. Ces expériences conjointes se poursuivent de proche en proche jusqu'à ce qu'une routine efficace soit trouvée. Elles constituent donc un processus d'adaptation orienté vers l'identification de la meilleure façon de faire les choses, un processus par essais-erreurs dans lequel le nombre d'expériences à réaliser dépend des résultats des différentes rétroactions.

Les expérimentations conjointes peuvent prendre deux formes, selon le mécanisme de création de connaissances qu'elles utilisent et le type de connaissance auquel ce mécanisme donne lieu au sein des coentreprises. On distingue d'abord la forme empirico-inductive dans laquelle la création de connaissances est fondée sur les échanges d'expériences qui ont lieu au cours des interactions sociales entre les individus provenant des entreprisesmères. Il s'agit d'une forme de création de connaissances qui privilégie l'action et l'efficience au détriment d'une réflexion profonde et d'une pensée logique qui vise la recherche de concepts d'application universelle. Par ailleurs, cette forme de création de connaissances améliore la qualité des connaissances tacites. La deuxième forme d'expérimentation conjointe est la forme rationnelledéductive dans laquelle la création de connaissances est fondée sur une logique rationnelle et donne lieu à de nouvelles connaissances explicites. Cette création de connaissances très répandue dans les pays occidentaux se fait par une combinaison de connaissances existantes. 
Les expérimentations conjointes peuvent prendre deux formes, selon le mécanisme de création de connaissances qu'elles utilisent et le type de connaissance auquel ce mécanisme donne lieu au sein des coentreprises. On distingue d'abord la forme empirico-inductive dans laquelle la création de connaissances est fondée sur les échanges d'expériences qui ont lieu au cours des interactions sociales entre les individus provenant des entreprises-mères. II s'agit d'une forme de création de connaissances qui privilégie l'action et l'efficience au détriment d'une réflexion profonde et d'une pensée logique qui vise la recherche de concepts d'application universelle. Par ailleurs, cette forme de création de connaissances améliore la qualité des connaissances tacites. La deuxième forme d'expérimentation conjointe est la forme rationnelle-déductive dans laquelle la création de connaissances est fondée sur une logique rationnelle et donne lieu à de nouvelles connaissances explicites. Cette création de connaissances très répandue dans les pays occidentaux se fait par une combinaison de connaissances existantes.

L'expérimentation empirico-inductive et l'expérimentation rationnelle-déductive ne constituent pas des modes de création de connaissances isolés et exclusifs l'un de l'autre. Au contraire, les deux modes de création de nouvelles connaissances s'opèrent simultanément en interagissant l'un sur l'autre. Il s'agit d'un phénomène de réflexion dans l'action - c'est-à-dire l'expérience -, un phénomène caractérisé par une interaction entre l'expérience et la rationalité.

La création conjointe de connaissances en vue d'un développement de compétences stratégiques est nécessaire aux entreprises qui recherchent une meilleure position compétitive. Toutefois, cette nécessité ne doit pas faire perdre de vue les effets pervers qu'elle peut produire. En effet, l'approfondissement continu et cohérent des compétences stratégiques, leur diffusion et leur exploitation renforcent les risques d'inertie de l'entreprise et peuvent bloquer sa capacité à innover. Il s'agit du «piège de surcompétences ${ }^{5} »$ : il se traduit par le fait qu'à force de perfectionner ses compétences pour mieux s'adapter à certaines niches de marché, l'entreprise peut devenir la victime de ses propres efforts de perfectionnement, puis- qu'elle peut se trouver incapable d'innover ou de s'adapter à d'autres niches de marché lorsque la conjoncture de son environnement le requiert. Pour éviter ce «piège des surcompétences », l'entreprise doit trouver un juste équilibre qui contrecarre les forces d'inertie et d'entropie naturelles. Autrement dit, elle doit faire un arbitrage judicieux entre une amélioration continue de ses compétences stratégiques et leur renouvellement. La création conjointe de connaissances en vue d'un développement de compétences stratégiques peut s'articuler autour de deux mécanismes importants, à savoir la transformation conjointe de connaissances et les expérimentations conjointes.

\section{La transformation conjointe des connaissances}

Le transfert et l'internalisation de connaissances ne sont pas les seules pratiques d'apprentissage qui peuvent permettre un développement de compétences stratégiques. La transformation conjointe des connaissances est l'une des pratiques d'apprentissage qui permet le développement de compétences stratégiques. En effet, le souci de la coentreprise de répondre adéquatement aux besoins de son marché l'amène à s'engager dans un processus d'adaptation des connaissances acquises des entreprises-mères. Ce processus qui doit se réaliser conjointement par les employés de la coentreprise provenant des entreprises-mères respectives peut lui permettre d'améliorer ses aptitudes ou procédés de fabrication et même dans certains cas de s'engager dans une innovation incrémentale.

Un tel processus se traduit évidemment par le développement de nouvelles habiletés spécifiques qui favorisent une amélioration de la position compétitive de la coentreprise. C'est la nature spécifique de ces habiletés qui leur confère un caractère stratégique. Elles reposent en effet sur des connaissances difficiles à imiter par les concurrents. La transformation des savoir-faire se traduit entre autres par les modes de conversion de connaissances que sont la socialisation, la combinaison, l'internalisation et l'externalisation. Ainsi, la coentreprise devra procéder à une transformation de connaissances soit par socialisation, c'est-à-dire une conversion de connaissances tacites en connaissances tacites, soit par combinaison, c'est-à-dire une conversion de connaissances explicites en connaissances explicites. Elle peut également procéder à une transformation de connaissances soit par internalisation, c'est-à-dire une conversion de connaissances explicites en connaissances tacites, soit par externalisation, 
c'est-à-dire une conversion de connaissances tacites en connaissances explicites.

Ces différents modes de conversion de connaissances ne se réalisent pas de manière isolée les unes des autres dans l'organisation. Au contraire, elles se réalisent de manière simultanée, ce qui enrichit l'apprentissage. En effet, la promotion de différentes connaissances organisationnelles, et plus précisément la diversité de la base d'apprentissage de l'organisation favorise le développement rapide de nouvelles habiletés spécifiques.

\section{Les expérimentations conjointes}

L'expérimentation est un mode d'apprentissage fondamental dans le développement de compétences stratégiques. Plus spécifiquement dans le cadre des coentreprises internationales, cette expérimentation est conjointe puisqu'elle résulte d'une collaboration entre les employés de la coentreprise provenant des entreprises-mères. L'expérimentation opère surtout par des essais-erreurs ou des combinaisons de connaissances et peut permettre un développement de compétences stratégiques au sein de la coentreprise, notamment à travers une amélioration continue de ses connaissances, ou encore à travers des innovations.

En effet, les essais-erreurs permettent à l'organisation d'identifier la meilleure façon de faire. Ils amènent l'organisation à accumuler des expériences qui peuvent l'aider à créer son propre processus incrémental de développement de connaissances technologiques en vue de réaliser une amélioration continue de ses produits ou procédés. Un tel processus est fondé sur les relations très étroites qu'entretiennent les employés et, de ce point de vue, aboutit à une ambiguïté causale qui rend les connaissances ainsi développées difficilement imitables par les concurrents. La coentreprise développe ainsi des compétences stratégiques puisque le caractère difficilement imitable des connaissances ainsi développées lui permet d'avoir ou de conserver des avantages compétitifs.

Les essais-erreurs peuvent être accompagnés d'une combinaison de savoir-faire. Dans ce cas, l'expérimentation implique une intégration d'habiletés diverses. Il s'agit bien entendu dans le cas des coentreprises, d'habiletés provenant des entreprises-mères, notamment à travers les individus qui les y représentent. Une telle intégration requiert que l'organisation redéfinisse son cadre de référence cognitive afin de pouvoir se mettre rapidement au rythme des nouvelles technologies ${ }^{5}$. L'expérimentation sous forme d'essais-erreurs et de combinaison de connaissances entraîne le développement d'une capacité rapide d'innovation au sein de la coentreprise, grâce à la création d'un nouveau schéma cognitif caractérisé par la dominance d'une nouvelle logique managériale.

L'apprentissage par expérimentations conjointes conduit à un développement de compétences stratégiques par une amélioration continue des produits ou procédés de la coentreprise, mais aussi et surtout par la création de nouveaux produits ou procédés, à partir d'un nouveau schéma de connaissances. En effet, l'amélioration continue et la création de nouveaux produits ou procédés permettent à la coentreprise de développer ou de maintenir une meilleure position compétitive.

Au total, l'apprentissage par expérimentations conjointes conduit à un développement de compétences stratégiques par une amélioration continue des produits ou procédés de la coentreprise, mais aussi et surtout par la création de nouveaux produits ou procédés, à partir d'un nouveau schéma de connaissances. En effet, l'amélioration continue et la création de nouveaux produits ou procédés permettent à la coentreprise de développer ou de maintenir une meilleure position compétitive.

\section{Les conditions de succès de l'apprentissage stratégique dans les coentreprises internationales}

Les conditions de succès de l'apprentissage constituent l'une des principales préoccupations des chercheurs qui travaillent sur le phénomène de l'apprentissage. Plus spécifiquement dans le cas des coentreprises internationales, certaines de ces conditions paraissent particulièrement importantes. Il s'agit de l'intention d'apprendre, de la capacité d'apprendre, des mécanismes de transfert des connaissances, d'une collaboration constructive et enfin d'une gestion des spécificités socioculturelles. L'intention d'apprendre suppose une grande détermination de la part des apprenants, mais aussi et surtout une valeur stratégique des connaissances transférées. La valeur stratégique de la connaissance constitue un déterminant qui peut influencer l'intensité du désir de la coentreprise de l'apprendre. 


\section{L'intention d'apprendre suppose une grande détermination de la part des apprenants, mais aussi et surtout une valeur stratégique des connaissances transférées. La valeur stratégique de la connaissance constitue un déterminant qui peut influencer l'intensité du désir de la coentreprise de l'apprendre.}

La capacité d'apprendre suppose que les apprenants de la coentreprise ont les qualifications requises pour accéder aux savoir-faire de l'entreprise-mère étrangère. En effet, il ne suffit pas de manifester une intention d'apprendre pour que l'apprentissage soit un succès; il faut aussi en avoir les capacités. Ceci est particulièrement vrai dans le contexte économique d'aujourd'hui, celui d'une économie de savoir marquée par une évolution prodigieuse du développement des nouvelles connaissances. La capacité d'apprendre est généralement fondée d'une part sur la qualification qui elle-même dépend de la formation et de l'expérience de travail, et d'autre part sur la réceptivité. Les mécanismes de transfert se traduisent par la mise en œuvre de programmes de formation ainsi que des détachements d'experts provenant des entreprises-mères pour assurer efficacement ces formations.

La plupart des coentreprises internationales ayant des objectifs d'apprentissage stratégique recourent à un détachement en leur sein des experts de l'entreprise-mère. Il ne peut en être autrement puisque ces experts détiennent les connaissances recherchées par la coentreprise. Néanmoins, un transfert réussi des connaissances d'une entreprise-mère étrangère à la coentreprise requiert bien évidemment une bonne collaboration et en particulier une relation de confiance entre techniciens locaux de la coentreprise et leurs homologues provenant de l'entreprise-mère étrangère. Le développement de cette relation de confiance se traduit par une transparence dans les échanges d'informations techniques, un esprit d'ouverture entre techniciens de la coentreprise et techniciens provenant de l'entreprise-mère et par une réalisation d'activités sociales par ces derniers.

Enfin, il est indispensable qu'au cours de l'apprentissage, les techniciens locaux de la coentreprise tiennent compte des spécificités socioculturelles de leurs homologues provenant de l'entreprise-mère étrangère et inversement.
En effet, les coentreprises sont généralement un cadre d'échanges interculturels qui, lorsqu'ils sont bien gérés, sont source de créativité. Au contraire, lorsqu'ils sont mal gérés ils peuvent ralentir, appauvrir ou même obstruer l'apprentissage de la coentreprise. Pour éviter ces difficultés, il est vivement souhaitable que la coentreprise offre à ses cadres qui seront impliqués dans le processus d'apprentissage avec les techniciens étrangers, un programme de préparation qui les sensibilise aux différences socioculturelles de ces derniers. De nombreuses grandes entreprises engagées dans des activités internationales offrent régulièrement ces programmes de préparation à leurs cadres avant de les affecter à l'étranger. Un des principaux avantages de cette préparation est qu'elle permet d'éviter les conflits à fond culturel. Si toutefois de tels conflits surviennent au cours de l'apprentissage, il est impérieux de les régler de manière pacifique.

\section{La plupart des coentreprises internationales ayant des objectifs d'apprentissage stratégique recourent à un détachement en leur sein des experts de l'entreprise-mère. II ne peut en être autrement puisque ces experts détiennent les connaissances recherchées par la coentreprise. Néanmoins, un transfert réussi des connaissances d'une entreprise-mère étrangère à la coentreprise requiert bien évidemment une bonne collaboration et en particulier une relation de confiance entre techniciens locaux de la coentreprise et leurs homologues provenant de l'entreprise-mère étrangère.}

\section{Conclusion}

L'apprentissage stratégique est l'un des moyens pouvant permettre à la coentreprise internationale d'avoir une meilleure position compétitive. En effet, il favorise un développement de nouvelles compétences qu'il est difficile pour les concurrents d'imiter ou de rattraper. Toutefois, l'apprentissage stratégique continue d'être un phénomène qui n'est pas bien connu, en particulier dans les coentreprises internationales. Et pourtant, la coentreprise internationale constitue l'une des formes de coopération interentreprises de plus en plus répandues, et l'apprentissage de savoir-faire nouveaux constitue dans de nombreux cas l'une des principales motivations qui justifie sa création. 
Il s'agit d'un apprentissage qui a lieu dans un contexte assez particulier puisque les partenaires à l'apprentissage, les entreprises-mères, collaborent selon une logique de conflit-coopération. En effet, dans ce type d'apprentissage, la volonté de coopérer avec son partenaire en vue d'avoir accès à son savoir-faire coexiste la plupart du temps avec des divergences qui sont malheureusement source de conflits. Quelques-unes de ces divergences proviennent des spécificités socioculturelles propres à chaque partenaire, ou encore des objectifs particuliers visés par les différents partenaires et qui parfois ne sont pas clairement exprimés.

\section{Notes et références}

1 Élie Virgile Chrysostome est professeur de management à la Faculté d'administration de l'Université de Moncton.

2 Huber, G.P. (1991). «Organizational Learning: The Contributing Process and The Literatures». Organization Science, vol. 2, n ${ }^{\circ} 1$, pp. 88-115.

3 Lei, D., M.A. Hitt et R. Bettis (1996). «Dynamic Core Competences Through Meta-Learning and Strategic Context». Journal of Management, vol. 22, $\mathrm{n}^{\circ}$ 4, pp. 549-569.

4 Reed, R. et R.J. DeFillippi (1990). «Causal Ambiguity, Barriers to Imitation and Sustaining a Competitive Advantage». Academy of Management Review, vol. 15, $\mathrm{n}^{\circ}$ 1, pp. 88-102.

5 Doz, Y. (1994). «Les Dilemmes de la Gestion du Renouvellement des Compétences clés ». Revue Française de Gestion, n 97, pp. 92-104. 